www.jmscr.igmpublication.org

Impact Factor (SJIF): 6.379

Index Copernicus Value: 71.58

ISSN (e)-2347-176x ISSN (p) 2455-0450

crossref DOI:_https://dx.doi.org/10.18535/jmscr/v6i4.62

Journal Of Medical Science And Clinical Research

IGM Publication

An Official Publication of IGM Publication

\title{
Assessing the validity and reliability of a questionnaire to identify existence of parental neglect in terms of developing feeding style and social activity in obese adult saudi population
}

\author{
Authors \\ Khurshid A Mattoo ${ }^{*}$, Mohd. Moaleem ${ }^{1}$, Mosa Shubayr ${ }^{2}$ \\ ${ }^{1}$ *Department of Prosthetic dental sciences, College of Dentistry, Jazan University, (KSA) \\ ${ }^{2}$ Meharry Medical College, Nashville, Tennessee (USA) \\ *Corresponding Author \\ Khurshid A Mattoo \\ Email:drkamattoo@rediffmail.com
}

\begin{abstract}
This study aimed to validate a questionnaire that was formulated to identify existence of parental neglect in regard to the feeding style and social activity in their overweight or obese children. The questionnaire is the result of three different surveys done over a period of time (prepilot, pilot and actual study). For negligence of feeding style, six domains were identified [responsibility, perception (5 item), feeding control (4 item), feeding culture (3 item), feeding, environment (6 item), feeding, monitoring (5 item) and feeding restrictions (5 items)] while social activity had a 9 item question. Validity was assessed and evaluated using average congruency percentage during the pilot study while before the actual study the I-CVI for items and for scale were determined. The content validity index for the item and for the scale were excellent with values ranging between 0.83 to 1. Internal reliability for individual questions (crohnbach alpha value of .734 to .915) and of each domain in relation to all questions and the negligence scale demonstrated excellent reliability.

Keywords: child maltreatment, research tools, index, measuring scale, child protection.
\end{abstract}

\section{Introduction}

Saudi Arabia covers 80 percent of the largest peninsula of the world, the Arabian peninsula. With less population distribution over vast areas and large resources of oil and natural gas, economic prosperity has changed the socioeconomic status of Saudis. ${ }^{1}$ Socioeconomic boost along with unfavourable weather conditions and unfavourable landscape prompts the population to stay indoors while enjoying the luxury of food and television. Practicing of such behaviour in families has given sudden and alarming rise in the prevalence of overweight and obesity (Ow/Ob) both among the children as well as adults. While between 1988 to 2005, the rise of overweight boys was observed from 3 to $25 \%,,^{2,3}$ national survey data of 2013 estimated about 35 to $40 \%$ of the adult population to be obese. ${ }^{4}$ Independent studies around the same time (2014-15) have reported 66-75\% of adults and $25-40 \%$ of children to be either overweight or obese in the Arab region. ${ }^{6}$

Although its multifactorial aetiology is hereditary in nature in $40-70 \%$ of cases, ${ }^{7}$ environmental influences play important role in the remaining cases, while exaggerating those that are genetic in nature. Among environmental influences, parent neglect (child neglect) has been found to havea major influence in both cause and prevention especially on adulthood obesity. ${ }^{8-12}$ The subject of child neglect is a socially sensitive issue in many 
conservative cultures and although happening for centuries, there is a dearth of studies where cultural norms suppress any form of open discussion on child and even elder maltreatment. Saudi Arabia has had a long history of harsh physical treatment of juveniles till 1990, after which there has been growing recognition of the scale of child abuse and neglect (CAN) problem. ${ }^{13}$ While in $2010,{ }^{14}$ the Saudi national family registry (NFSR) reported 292 (43\% neglect) cases from 38 hospital based child protection centres, the number of reported instances rose to 1,450 in $2012^{15}$ ( $35 \%$ being neglect). After 2012 there has been no further reportsof the government. Whether underreporting or not reporting, the fact remains that parent neglect exists and could be a dynamic factor in the development of the alarming prevalence of obesity in the region. Although there are different methods to determine child neglect, the most practical being to observe the home environment of the child. Since cultural, religious and social restrictions do not allow the use of such methods in conservative cultures, therefore, one of the methods to establish parental neglect (in terms of feeding and social activity) is by using a questionnaire as described in a study previously. ${ }^{16}$ This study is a further continuation of its previous study and in this study, we assess the validity and reliability of a refined questionnaire which can be used as a tool to measure parental negligence in terms of feeding style and social activity.

\section{Materials and Methods}

This study was conducted after appropriate ethical approval from university ethicscommittee, that conducts research in accordance with Helsinki declaration. ${ }^{17}$ Informed consent was obtained wherever appropriate, from all subjects and their parents or legal guardians. Questionnaire used in this study is illustrated in Appendix 1(after establishing validity and reliability). The questionnaire was the outcome of multiple pre-pilot and pilot surveys that were developed on the basis of focus group discusssions. ${ }^{16}$ Parental neglect in terms of feeding had six domains (responsibility perception, feeding control, feeding culture, feeding environment, monitoring and feeding restrictions) while social activity had one domain that contained a set of 9 questions.

Participants: The results are based on multiple surveys that were held at various times. However, for assessing the validity and reliability, the tool was surveyed among 90 adult obesesubjects (18 -30 years).In establishing the domains of feeding styles and social activity, random subjects were taken irrespective of their Body mass index during a pilot study. ${ }^{16}$ For dual moderator group discussions, recognized social members of the region participated who inhabited the region for generations and possessed thorough knowledge of a population's life style. For test retest reliability, subjects were identified through convenience sampling with criteria of having a BMI above 25, subjects living with their parents both living since childhood and had no evident clinical systemic disorder that could be a cause of their being overweight. The same group of subjects was given the same questionnaire at different period of time (3 months apart).

Validity: The questionnaire was evaluated by a committee of six experts(1 social psychologist, 1 epidemiologist, 2 nutritionists, 1 behavioural analyst and 1 general physician). All members possessed relevant necessary experience in their respective fields. The experts evaluated the validity by using the method of average congruency percentage (ACP) in the first pre-pilot and a pilot study. ${ }^{18}$ Evaluation was done for readability, lucidity and viability of wording and layout and style. Domains of parental neglect were inspired by focus group dual moderator discussions done before and during pre pilot and a pilot study. The questionnairewas then translated into Arabic language (local) and then translated back into English according to the 'translation/back -translation method'19 by two bilinguals having experience of more than 5 years in the respective field. Questions were accustomed according to the socio-cultural conditions and available sports facilities in the region. Content validity was evaluated using Item - content validity index (I-CVI) and scale - content validity index (S- 
CVI). ${ }^{18}$ For each domain (construct) the interrelationship among items that share sufficient variation was analysed. All unrelated items were eliminated. To study concurrent validity, the questionnaire was surveyed among the parents of obese subjects and parents of non obese subjects. The parent neglect score was calculated for each domain and a combination of six scores for determining the negligence in terms of feeding style. While for social activity, the scores were determined in one general domain.

Reliability: To determine stability of the questionnaire in determining parent neglect, test retest method was used during which the test was administered twice to the same participants at different times (3 month interval). All subjects were verified environmentally modulated obese subjects. For internal consistency of the questionnaire statistical tests were used (cronbach alpha). Inter rater reliability tests were done to ensure equivalence. Parent- child reliability was evaluated based on the parent neglect questionnaire (PNQ) filled by both the child and parent, independently of each other.

Data analysis: For determining the I-CVI, all six judges rated each question/item of the questionnaire in terms of its relevance to the underlying construct to a 4 point ordinal scale (1: not relevant, 2: somewhat relevant,3: quite relevant and 4: highly relevant). The I-CVI was computed for each item as the number of experts giving a rating of either 3 or 4 divided by total no of experts. An I-CVI higher than 0.8 was considered as significant.Interitem reliability was calculated by cronbach alpha $(\alpha)$ for which a value of 0.70 was considered as significant.

\section{Results}

An overview of content validity is represented in Table 1 for both individual items and the parent neglect scale. A total of 13 individual items demonstrated highest index score of $1(38 \%)$ while 24 items showed an index value of 0.8 (62\%). About 19 judgements were highly relevant on the assessment scale while the rest were quite relevant. For parent neglect scale, the domain of responsibility perception and feeding culture showed highest index values of 1 while four domains had S-CVI score of 0.83 (feeding control, feeding, environment, monitoring, and feeding restrictions) with social activeness scoreindex of 0.67.Reliability of individual items and various domains of parent neglect scale at the first test and retest using crohnbach alpha are illustrated in Table 2. The domain of responsibility, perception $(0.900$ test -0.816 retest $)$ and feeding restrictions $(0.770$ test -0.812 retest) where the two domains that showed non significant variation in values between the first test and retest. However, both values in the test and retest remained significant.

\begin{tabular}{|c|c|c|c|c|c|c|c|c|}
\hline \multicolumn{9}{|c|}{$\begin{array}{l}\text { Table 1: Content validity index of individual items (I-CVI) and content validity indes of } \\
\text { the scale (S-CVI) for parent neglect questionnaire (PNQ) }\end{array}$} \\
\hline \multicolumn{9}{|c|}{ Content validity index-item (I-CVI) scores for the parent neglect questionnaire } \\
\hline S. no & Item & Ex 1 & Ex 2 & Ex 3 & Ex 4 & Ex 5 & Ex 6 & I-CVI \\
\hline 1) & $1,3,6,8,10,12$ & 4 & 4 & 4 & 4 & 4 & 4 & 1 \\
\hline 2) & $19,26,31,36$ & 4 & 4 & 4 & 4 & 3 & 4 & .83 \\
\hline 3) & $4,5,13,15.17,18$ & 4 & 3 & 3 & 3 & 3 & 3 & .83 \\
\hline 4) & $2,7,9,20,22,33,35,37$ & 3 & 3 & 3 & 3 & 4 & 3 & .83 \\
\hline 5) & $11,16,21,23,24,29,30$ & 3 & 3 & 3 & 3 & 3 & 3 & 1 \\
\hline 6) & $25,27,28$ & 3 & 4 & 3 & 3 & 3 & 3 & .83 \\
\hline 7) & $14,37,32,34$ & 4 & 4 & 4 & 4 & 3 & 4 & .83 \\
\hline \multicolumn{9}{|c|}{ Content validity index-scale (S-CVI) scores for the parent neglect domains } \\
\hline & Domain & Expert 1 & Expert 2 & Expert 3 & Expert 4 & Expert 5 & Expert 6 & S-CVI \\
\hline 1). & Responsibility perception & 3 & 3 & 3 & 3 & 3 & 3 & 1 \\
\hline 2). & Feeding control & 4 & 4 & 4 & 4 & 3 & 4 & .83 \\
\hline 3). & Feeding culture & 4 & 4 & 4 & 4 & 4 & 4 & 1 \\
\hline 4). & Feeding environment & 3 & 3 & 3 & 3 & 4 & 3 & .83 \\
\hline 5). & Monitoring feeding & 3 & 3 & 3 & 3 & 3 & 4 & .83 \\
\hline 6$)$. & Feeding restrictions & 4 & 4 & 4 & 4 & 3 & 4 & .83 \\
\hline 7). & Social activeness & 4 & 3 & 3 & 3 & 4 & 3 & .67 \\
\hline
\end{tabular}




\begin{tabular}{|c|c|c|c|c|}
\hline \multicolumn{5}{|c|}{$\begin{array}{l}\text { Table } 2 \text { Internal consistency (reliability) values of parental characteristics in } \\
\text { term of feeding style and social activity among saudi obese population - } \\
\text { results of a pilot study }\end{array}$} \\
\hline S.no & Parenting characteristics & $\begin{array}{c}\text { Internal } \\
\text { reliability } \\
(\text { Cronbach } \alpha) \\
(\mathbf{n}=90)\end{array}$ & $\begin{array}{l}\text { Cronbach alpha } \\
\text { based on } \\
\text { standardized items }{ }^{\mathrm{a}}\end{array}$ & Test retest reliability \\
\hline 1. & Responsibility perception & 0.900 & 0.823 & 0.816 \\
\hline 2. & Feeding control & 0.819 & 0.784 & 0.802 \\
\hline 3. & Feeding culture & 0.883 & 0.721 & 0.812 \\
\hline 4. & Feeding environment & 0.915 & 0.745 & 0.902 \\
\hline 5. & Monitoring feeding & 0.734 & 0.739 & 0.798 \\
\hline 6. & Feeding restrictions & 0.770 & 0.819 & 0.812 \\
\hline 7. & Social activeness & 0.795 & 0.689 & 0.754 \\
\hline \multicolumn{2}{|c|}{$\begin{array}{l}\text { Overall questionnaire ( } 34 \text { items - one } \\
\text { item deleted) }\end{array}$} & 0.857 & 0.794 & 0.824 \\
\hline
\end{tabular}

\section{Discussion}

Neglect is a circumstance where inaction of the parents deprives the child of his basic right. The neglect observed in children is basically either in their care or in their supervision. Social, cultural and more important religious limitations in middle east countries do not allow to observe and identify neglect of a child by observing his home environment as non family members have restrictions to a private house in many of middle east and south east cultures. However, since obesity has a very strong relation with parental neglect, this study is an attempt to prepare a scaled questionnaire that would identify the existence of parental neglect in individuals who are obese. For this study, we evaluated the reliability and validity of a saudi version of a PNQ (parent neglect questionnaire, although neglect is related to only feeding style and social activity). This study discusses the following:

Questionnaire: A questionnaire that would assess the existence of parental neglect has been formulated for this study. Because the construct neglect has highly varied domains, therefore this question focuses only parent neglect in terms of feeding style and social activity, both variables having significant impact on obesity outcome especially adulthood obesity. The questionnaire (Appendix 1) is the outcome of multiple attempts and refinement that is not reflected in this study. The questions/ items presented in the questionnaire are those who were highly valid and reliable although further studies need to be conducted on its application in different cultures. The questions are based on modification of

Home Observation for Measurement of the Environment (HOME), ${ }^{20,21}$ the home environment questionnaire (HEQ), ${ }^{22}$ comprehensive feeding practices questionnaire (CFPQ) ${ }^{23}$ parenting styles and dimensions questionnaire (PSDQ), ${ }^{24}$ food frequency questionnaire (FFQ) ${ }^{25}$ and the validated childhood experience of care and abuse (CECA.Q). ${ }^{26}$ Since these questionnaires are long and require knowledgable parents, the questionnaire presented in our study is short with simple language while maintaining the required internal consistency values. The sensitive part of the questionnaire is its drawback also and it is related with the reverse coding for questions that are negatively keyed items. Extreme caution must be exercised while statistical determination in such cases. To assess parent neglect, it is important to use instruments that are non invasive especially in conservative and religious cultures. Moreover, the tool is easy to use, time saving and can be used in children as well as adults. To our knowledge, there are no such questionnaires that have been attempted to assess parental negligence of overweight and obese adults. The usefulness lies in its ability to predict obesity risk in young children whose feeding and social activity has been neglected by their parents. The CECA.Q by Bifulco et al. is a self reported questionnaire whose focus is to assess lack of parental care in terms of neglect and antipathy, physical and sexual abuse before age 17. The drawbacks of the questionnaire are that it is self 
reported and response bias will be present in such instruments of measurement. Moreover, reliability and validity can differ based on ethnicity, geography and lifestyle subjects. Social activity assessment in this questionnaire only relates to the responsibility of parents to cultivate such habits in their children. Similarly the same holds true for feeding style also.
Parent neglect scale: To develop the weight of the responses given by the subjects, the level of each item was determined by the following formula: (highest point in likert scale - lowest point in likert scale)/ the number of levels used. ${ }^{27}$ The outcome of using this formula is that one could assess the overall construct of negligence in terms of statistically and position the subject relative to the scale (Table 3).

\begin{tabular}{|c|c|c|c|c|}
\hline \multicolumn{5}{|c|}{ Table 3: Scoring of domains of parent neglect } \\
\hline \multicolumn{5}{|c|}{ Ranges of parent neglect individual domain scores } \\
\hline $1-1.80$ & $1.81-2.60$ & $\begin{array}{l}2.61 \quad \text { to } \\
3.40\end{array}$ & $3.41-4.20$ & 4.21 to 5 \\
\hline $\begin{array}{l}\text { Very } \\
\text { negligent }\end{array}$ & Negligent & Neutral & Diligent & $\begin{array}{l}\text { Very } \\
\text { diligent }\end{array}$ \\
\hline \multicolumn{5}{|c|}{ Range of parent neglect questionnaire (total of 6 domains) } \\
\hline $6-10.8$ & $\begin{array}{l}10.86- \\
15.6\end{array}$ & $\begin{array}{l}15.66- \\
20.4\end{array}$ & $20.46-25.2$ & $25.26-30$ \\
\hline $\begin{array}{l}\text { Very } \\
\text { Negligent }\end{array}$ & Negligent & Neutral & Diligent & $\begin{array}{l}\text { Very } \\
\text { diligent }\end{array}$ \\
\hline
\end{tabular}

\section{Conclusion}

This is the first validation and reliability study of saudi version of the parent neglect questionnaire in terms of developing feeding habits and social activity among their children. The results clearly indicate the instrument is reliable and valid for adults in the age of 18 to 30 years. The questionnaire can be considered as very useful in clinical practice to identify existence of parental neglect in these two domains in overweight and obese subjects. Such assessments are useful both at individual and community level to improve personalized intervention programs and new health policies.

\section{Competing interests}

The authors declare that they have no competing interest in publishing the results of the study

\section{Acknowledgements}

This study in totality is supported by a grant fund from the $7^{\text {th }}$ research program of Deanship of Scientific Research, College of Dentistry, Jazan
University. We also thank all the participating subjects and their parents for their willingness to distribute information that is personal and sensitive.

\section{References}

1. Al-Hazzaa HM. Prevalence and trends in obesity among school boys in Central Saudi Arabia between 1988 and 2005. Saudi Med J 2007;28:1569-74

2. Mirmiran P, Sherafat-Kazemzadeh R, JalaliFarahani S, Azizi F. Childhood obesity in the Middle East: a review. East Mediterr Health J. 2010 Sep; 16 (9): 1009-17.

3. Ng SW, Zaghloul S, Ali HI, Harrison G, Popkin BM. The prevalence and trends of overweight, obesity and nutrition-related noncommunicable diseases in the Arabian Gulf States. Obes Rev. 2011 Jan; 12 (1): 1-13.

4. Institute of Health Metrics and Evaluation (IHME) data visualisation tool, Overweight and obesity patterns by country. Available at: http:// vizhub. Health data. Org/obesity 
5. Abdul Rahim HF, SibalA, KhaderY, Hwalla N, FadhilL et al. Health in the Arab world: a view from within 2 noncommunicable diseases in the Arab world. Lancet 2014;383:356-67

6. AlhaqwiA, AlnasirM, Ahmed N, MasaudiE, AlotaibiS, et al. Obesity and overweight in a major family practice centre, central region, saudi Arabia. Saudi J Obes 2015;3:12

7. Silventoinen K, Rokholm B, Kaprio J and Sorensen TI. The genetic and environmental influences on childhood obesity: a systematic review of twin and adoption studies. International Journal of Obesity (Lond). 2010; 34(1): 29-40.

8. Garrahan SM, Eichner AW. Tipping the Scale: A Place for Childhood Obesity in the Evolving Legal Framework of Child Abuse and Neglect. Yale Journal of Health Policy, Law, and Ethics, 2012;12 (2): 342-48

9. Gilbert R, Widom GR, Browne K, Fergusson $\mathrm{D}$, Webb $\mathrm{E}$ and Janson S. Burden and consequences of child maltreatment in highincome countries. Lancet, 2009; 373: 68-81

10. Christoffel KK and Forsyth BW. Mirror image of environmental deprivation: Severe childhood obesity of psychosocial origin. Child Abuse \& Neglect, 1989; 13: 249-256

11. Noll JG, Zeller MH, Trickett PK and Putnam FW. Obesity risk for female victims of childhood sexual abuse: A prospective study. Pediatrics; 2007; 120 (1): e61-7

12. Lissau I, Sorensen TI. Parental neglect during childhood and increased risk of obesity in young adulthood. Lancet 1994 Feb 5;343 (8893): 324-7

13. Al-Eissa YA (1998) Child abuse and neglect in Saudi Arabia: What are we doing and where do we stand? Ann Saudi Med 18: 105-106.

14. Health Ministry (2010). The National Family Safety Registry Report.

15. Health Ministry (2012). The National Family Safety Registry Report.

16. Mattoo KA, Moaleem M, Shubayr M. An analytical survey to define the dimensions of a scaled questionnaire that would determine the existence of parental neglect in environmentally modulated obese subjects - A pre - pilot and pilot study. Int. Journal of Medical Research and Pharmaceutical Sciences, 2018;5 (3): 1-10

17. World Medical Association. Declaration of Helsinki: Ethical Principles for Medical Research Involving Human Subjects. JAMA. 2013; 310(20): 2191-94

18. Polit DF, Cheryl Tatano Beck. The Content Validity Index: Are You Sure You Know What's Being Reported? Critique and Recommendations. Res Nurs Health. 2006;29:489-97

19. Brislin RW: Back-translation of cross-cultural research. J Cross Cult Psychol 1970, 1:185-216

20. Caldwell BM\& BradleyRH. Home observation and measurement of the environment. Little Rock: University of Arkansas Press.1978

21. Leventhal T, Senior-O'Hagan MB, BrooksGunn J, BingenheimerJB and Earls FJ. The homelife interview about the project on human development in Chicago neighbourhoods: Assessment of parenting and home environment for 3- to 15-year-olds. Parenting: Science \& Practice, 2004; 4: 211-241

22. Laing JA \&SinesJO. The Home Environment Questionnaire: An instrument for assessing several behavioural relevant dimensions of children's environments. Journal of Pediatric Psychology, 1982; 7: 425-449.

23. Musher-Eizenman D, Holub S. Comprehensive Feeding Practices Questionnaire: validation of a new measure of parental feeding practices. $\mathbf{J}$ Pediatr Psychol. 2007;32(8):960-72.

24. Sherman R, Fredman N. Handbook of measurements for marriage and family therapy. Routledge; 2013

25. Mirmiran P, Esfahani FH, Mehrabi Y, Hedayati M, Azizi F. Reliability and relative validity of an FFQ for nutrients in the Tehran lipid and glucose study. Public Health Nutr. 2010;13(5):654-62.

26. Bifulco A, Bernazzani O, Moran PM, Jacobs C. The childhood experience of care and abuse questionnaire (CECA.Q): validation in a 
community series. Br J ClinPsychol 2005;44/Pt

):563-81.

27. Dawes J. Do Data Characteristics Change

According to the number of scale points used?
An experiment using 5-point, 7-point and 10point scales". International Journal of Market Research, 2008; 50 (1): 61-77.

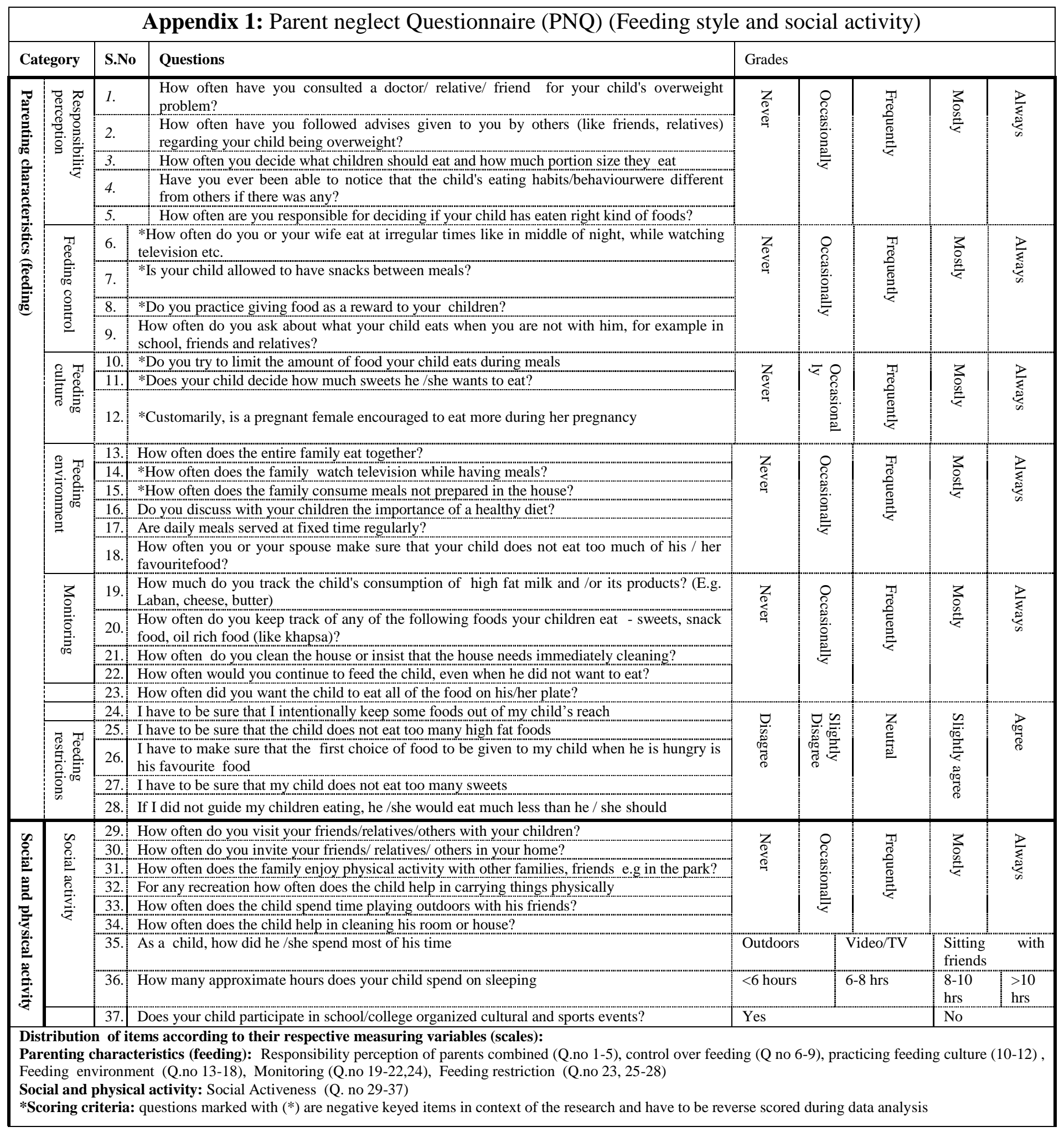

